\title{
Examining the Hofmeister Series through Activation Energies: Water Diffusion in Aqueous Alkali-Halide Solutions
}

\author{
Ashley K. Borkowski, Zeke A. Piskulich, and Ward H. Thompson* \\ Department of Chemistry, University of Kansas, Lawrence, KS 66045, USA \\ E-mail: wthompson@ku.edu
}

\section{Abstract}

The effect of ions on the properties of aqueous solutions is often categorized in terms of the Hofmeister series that ranks them from chaotropes ("structure-breakers"), which weaken the surrounding hydrogen-bond network to kosmotropes ("structure-makers"), which enhance it. Here, we investigate the Hofmeister series in $\sim 1 \mathrm{M}$ sodium-halide solutions using molecular dynamics simulations to calculate the effect of the identity and proximity of the halide anion on both the water diffusion coefficient and its activation energy. A recently developed method for calculating the activation energy from a single temperature simulation is used, which also permits a rigorous decomposition into contributions from different interactions and motions. The mechanisms of the salt effects on the water dynamics are explored by separately considering water molecules based on their location relative to the ions. The results show that water diffusion is accelerated moving down the halide group from $\mathrm{F}^{-}$to $\mathrm{I}^{-}$. The behavior of the diffusion activation energy, $E_{a}$, is more complex, indicating a significant role for entropic effects. However, water molecules in the first or second solvation shell of an ion exhibit a decrease in $E_{a}$ moving down the halide series and $\mathrm{Na}^{+}$ exhibits a larger effect than any of the anions. The $E_{a}$ for water molecules within the second solvation shell of an ion are modest, indicating a short-ranged nature of the ion influence.

\section{Introduction}

The Hofmeister series classifies ions based on their effect on the salting in and out of proteins. Since its development, this series has been explored in great detail, often with a particular focus on its broader implications for the effects of ions on the structure and dynamics of water in both biological and non-biological milieus. ${ }^{1121}$ In particular, ions in aqueous solution have been categorized based on their disruption or enhancement of the surrounding hydrogen-bonding (H-bonding) network as chaotropes ("structure-breakers") or kosmotropes ("structure-makers"), respectively. 117

A number of studies have shown that the Hofmeister series ordering does not hold for all properties, complicating the structure-making and rev-breaking interpretation. In fact, in some cases a "reverse" Hofmeister series is observed ${ }^{809}$ There is an ongoing debate over the nature of the effect of ion identity. This includes, the length scale over which ions impact the solvent structure and dynamics, $13-18$ the differences between anion and cation effects, $\frac{61311411821}{1}$ the relationship between between structure and dynamics,, 31416122223 and the relative roles of energetic and entropic driving forces. 31411612122124

An interesting issue in understanding the effect of ions on solvent properties is the differing 
roles of cations and anions. In experiments, it can be challenging to separate the influence of the two, though some spectroscopic approaches have been exploited.11 14 Molecular dynamics (MD) simulation studies of ionic solutions have reported a stronger effect on the dynamics of the surrounding solvent molecules due to the anion compared to the cation. ${ }^{6 / 13[18 \mid 21}$ In water and other H-bonding solvents, the asymmetry of the anion and cation solvation structure is a natural driving force of this difference, but the specific mechanisms that connect it to the dynamical properties are still being elucidated.

It has long been known that liquid structure and dynamics are closely related; disruptions in solvent structure lead to changes in solvent and solute dynamics. Dynamical timescales, including $\mathrm{OH}$ reorientation time, water diffusion coefficients, viscosity, and terahertz and infrared spectroscopic probes, often vary significantly with even relatively minor changes in the liquid structure. Understanding these ion effects on dynamics are key to unraveling the origins and interpretations of the Hofmeister series.

Diffusion coefficients have been found to provide a sensitive measure of the solvent response to ions with differing identities and concentrations. $\frac{120124}{33}$ They have the additional advantage that they are readily accessible in both measurements, primarily based on nuclear mag-

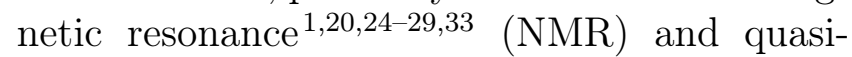
elastic neutron scattering, 34 (QENS), and MD

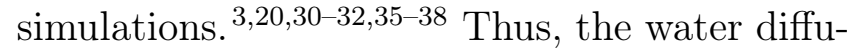
sion coefficient has been of significant interest in the case of aqueous salt solutions. While many of the trends are now clearly established, a complete picture of the underlying driving forces is not yet available; interesting, sometimes nonintuitive, behavior as a function of salt identity and concentration has been reported.20130 33

The self-diffusion coefficient of neat water has been extensively measured, $39 \sqrt{44}$ serving as an accurate reference for the influence of ions in electrolyte solutions. This effect is often expressed as $D / D_{0}$ where $D_{0}$ represents the neat water value. Some of the earliest NMR measurements of ionic solutions were reported by McCall and Douglass, 24 who considered nearly thirty different salts at varying concentrations, including the $\mathrm{NaCl}, \mathrm{NaBr}$, and $\mathrm{NaI}$ solutions considered in this work. Endom et al. likewise carried out an extensive study of water diffusion (and other dynamics) in various salt solutions as a function of concentration and temperature. ${ }^{25}$ Both studies found that $D$ was smaller than that in neat water $\left(D / D_{0}<1\right)$ for all the $\mathrm{NaCl}, \mathrm{NaBr}$, and $\mathrm{NaI}$ salts with the effect largest for $\mathrm{Cl}^{-}$and smallest for $\mathrm{I}^{-}$.

In this Paper, we present calculations of the water self-diffusion coefficient, $D$, in $\sim 1 \mathrm{M}$ sodium-halide solutions to gain insight into the effects on water dynamics at a moderate concentration that has been frequently studied $\frac{8|16| 20|24| 30 \mid 45}{4}$ and permits exploration of water dynamics in a diverse range of ionic environments. The aim is to better understand the Hofmeister series and associated classifications. In addition to the diffusion coefficient, we directly calculate the activation energy from simulations at a single temperature using the recently developed fluctuation theory for dynamics. $46[49]$ This approach also provides a rigorous decomposition of the activation energy associated with different motions and interactions, e.g., kinetic, Lennard-Jones, and electrostatic energies. The particular effects of the different ions in the sodium-halide solutions are elucidated by separately examining the behavior of water molecules based on their location (defined by solvation shells) relative to the ions present.

\section{Theory}

\section{Diffusion Coefficients and Activa- tion Energies}

The water diffusion coefficient can be obtained from MD simulations by calculating the meansquared displacement (MSD),

$$
M S D(t)=\frac{1}{N} \sum_{j=1}^{N}\left\langle\left|\vec{r}_{j}(t)-\vec{r}_{j}(0)\right|^{2}\right\rangle .
$$

Here $\vec{r}_{j}(t)$ is the oxygen atom position of the $j^{\text {th }}$ water molecule at time $t, N$ is the number of water molecules, and $\langle\cdot\rangle$ represents a thermal average within the canonical ensemble. At 
longer times $M S D(t)$ is linear in time with a slope proportional to $D$ as

$$
D=\lim _{t \rightarrow \infty} \frac{M S D(t)}{6 t} .
$$

In practice, $D$ is determined from the slope of a linear fit to $M S D(t)$ over a time interval.

A central focus of this Paper is the examination of the activation energy of the water diffusion coefficient,

$$
E_{a}=-\frac{\partial \ln D}{\partial \beta}
$$

where $\beta=1 / k_{B} T$. This activation energy provides important insight into the barrier underlying the diffusion process. While $E_{a}$ is typically obtained from an Arrhenius analysis, it is well established that water dynamics, including diffusion, 49 51 exhibits significant nonArrhenius behavior. The present work makes use of a recently developed method, derived from the application of fluctuation theory to dynamics, that has been demonstrated for calculating diffusion coefficient activation energies. $\underline{46} \underline{49}$

Specifically, we have previously shown that the derivative of the $M S D(t)$ with respect to inverse temperature $(\beta)$ is given by 47

$$
\begin{aligned}
\frac{\partial M S D(t)}{\partial \beta} & =-\frac{1}{N} \sum_{j=1}^{N}\left\langle\delta H(0)\left|\vec{r}_{j}(t)-\vec{r}_{j}(0)\right|^{2}\right\rangle \\
& \equiv-M S D_{H}(t)
\end{aligned}
$$

where $\delta H(0)=H(0)-\langle H\rangle$ is the fluctuation in the total system energy, $H$, at time $t=0$. Thus, the temperature dependence of $D$ is calculated from the correlation of the instantaneous fluctuations of the energy with the diffusive motion of the water molecules as measured by the MSD. It is then straightforward to show that the activation energy of $D$ can be written as,

$$
E_{a}=\frac{\lim _{t \rightarrow \infty} M S D_{H}(t)}{\lim _{t \rightarrow \infty} M S D(t)} .
$$

Note that $M S D(t)$ and $M S D_{H}(t)$ are both time correlation functions that can be com- puted from simulations at a single temperature. As opposed to the numerical derivative used in an Arrhenius analysis, eq. 5 is an analytical derivative. In practice in the present work, we calculate $E_{a}$ from the ratio of the slopes of $M S D_{H}(t)$ and $M S D(t)$ over the time interval 5-20 ps.

This approach for calculating the activation energy also provides important, otherwise unavailable mechanistic information. ${ }^{47+49|52| 53}$ In particular, the total energy can be decomposed in numerous physically meaningful ways. In the context of water diffusion in electrolyte solutions, a useful example is

$$
\delta H(0)=\delta K E(0)+\delta V_{L J}(0)+\delta V_{\text {Coul }}(0),
$$

where $K E$ is the kinetic energy and $V_{L J}$ and $V_{C o u l}$ are the Lennard-Jones and Coulombic potential energies. Substituting this for $\delta H(0)$ in eqs. 4 and 5 separates the total diffusion coefficient activation energy into contributions from these motions and interactions as

$$
E_{a}=E_{a, K E}+E_{a, L J}+E_{a, C o u l},
$$

with, for example,

$$
E_{a, \text { Coul }}=\frac{\lim _{t \rightarrow \infty} M S D_{\text {Coul }}(t)}{\lim _{t \rightarrow \infty} M S D(t)},
$$

where $M S D_{\text {Coul }}(t)$ is $M S D_{H}(t)$ defined in eq. 4 with $\delta H(0)$ replaced by $\delta V_{\text {Coul }}(0)$.

The physical interpretation of these contributions to the activation energy is provided by Tolman's perspective. ${ }^{48 \mid 54} 56$ Tolman showed that the activation energy for a chemical reaction is equal to the difference in the average energy of reacting species minus the average energy of the reactants. In other words, $E_{a}$ is the energy required to overcome the barrier to reaction (rather than just the height of the barrier itself). As such, the activation energy can be subdivided into different components of the total energy needed to pass over the barrier. For water diffusion, the activation energy is then the average energy of water molecules that diffuse (i.e., surmount the barrier to diffusion) minus the average energy of all water 
molecules. From this viewpoint, the contribution $E_{a, \text { Coul }}$ is the average electrostatic energy of water molecules that diffuse minus the average electrostatic energy of all water molecules. In this way, the decomposition in eq. 7 provides insight into which components of the system's energy most effectively accelerate (or decelerate) diffusion.

\section{Categorization of the Water Molecules}

To gain insight into the specific effect of the sodium and halide ions, each water molecule within a simulation is categorized - at every time origin used in the calculation of the diffusive dynamics, i.e., $M S D(t)$ and $M S D_{X}(t)-$ according to its location with respect to the ion solvation shells for both the cation and anion. We classify each water molecule according to whether its oxygen atom lies within the $1^{\text {st }}$ or $2^{\text {nd }}$ solvation shell of a cation, anion, a combination of two such solvation shell locations based on the specific ions involved, a combination of three (or more) solvation shells irrespective of the ions involved, or outside any such solvation shells. Fig. 1 provides a simplified illustration of how the water molecules are characterized in the case of an $\mathrm{NaCl}$ solution. This scheme results in a total of thirteen separate water populations that are defined in Table 1 .

The cutoff distances used to determine residence in the solvation shells were obtained from the minimums in the oxygen-ion radial distribution functions (RDFs) shown in Fig. 2a-e. Only the $\mathrm{NaF}$ results for $g_{O N a}(r)$ are shown in Fig. 27 as the RDF is nearly independent of the halide (see Fig. S1) and nearly the same cutoff distances for $\mathrm{Na}^{+}$solvation shells are used in all cases (see Table S2). Fig. 2b-e illustrates that descending the halide series from $\mathrm{F}^{-}$to $\mathrm{I}^{-}$, the $1^{\text {st }}$ and $2^{\text {nd }}$ solvation shell peaks flatten and broaden. This leads to changes in solvation shell cuttoffs; the values used are indicated in Fig. 2 by dashed lines and listed in Table S2.

For each category of water, the diffusion coefficient, corresponding activation energy, and activation energy components (i.e., associated with the kinetic, Lennard-Jones, and Coulom-

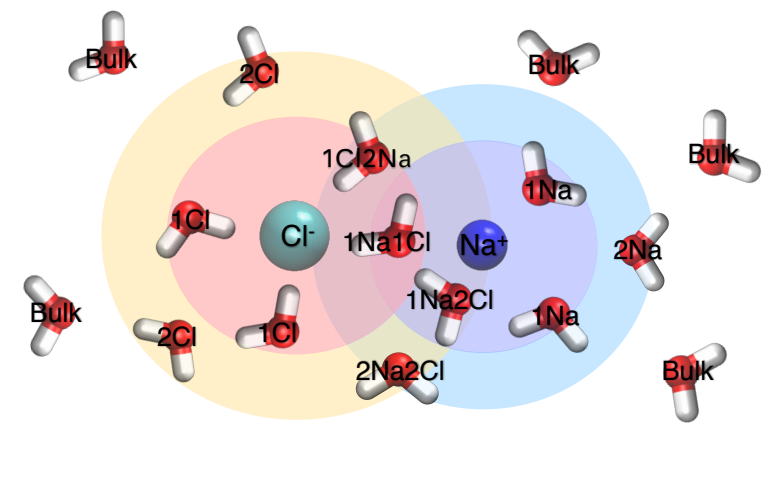

Figure 1: Schematic illustration for an $\mathrm{NaCl}$ solution demonstrating the populations of water molecules surrounding the ion pairs using the notation from Table 1

bic energies) are calculated to gain a mechanistic understanding of the ion effect. Namely, the mean-squared displacement can be rewritten as

$$
\begin{aligned}
M S D(t) & =\sum_{\alpha=1}^{13} f_{\alpha} \frac{1}{N_{\alpha}} \sum_{j=1}^{N_{\alpha}}\left\langle\left|\vec{r}_{j}(t)-\vec{r}_{j}(0)\right|^{2}\right\rangle \\
& =\sum_{\alpha=1}^{13} f_{\alpha} M S D_{\alpha}(t)
\end{aligned}
$$

where $\alpha$ labels the categories of water molecules based on solvation shell listed in Table 1, each consisting of $N_{\alpha}$ molecules and representing the fraction $f_{\alpha}=N_{\alpha} / N$ of all water molecules. An analogous expression can be written for the water diffusion coefficient,

$$
D=\sum_{\alpha=1}^{13} f_{\alpha} D_{\alpha}
$$

where $D_{\alpha}$ is determined from eq. 2 with $M S D(t)$ replaced by $M S D_{\alpha}(t)$, i.e., from the slope of $M S D_{\alpha}(t)$ at long times. The activation energy associated with the water diffusion coefficient is likewise additive, $E_{a}=\sum_{\alpha} f_{\alpha} E_{a, \alpha}$.

\section{Entropic Effects}

It is also interesting to estimate the entropic effects influencing the diffusion coefficient. One 
Table 1: Definitions of the categories used to denote locations of water molecules relative to sodium, $\mathrm{Na}^{+}$, and halide, $\mathrm{X}^{-}$, ions.

\begin{tabular}{|c|c|}
\hline Category & Definition \\
\hline All & All water molecules \\
\hline $1 \mathrm{X}$ & $1^{\text {st }}$ solvation shell of halide $\mathrm{X}^{-}$ \\
\hline $1 \mathrm{Na}$ & $1^{\text {st }}$ solvation shell of $\mathrm{Na}^{+}$ \\
\hline $2 \mathrm{X}$ & $2^{\text {nd }}$ solvation shell of halide $\mathrm{X}^{-}$ \\
\hline $2 \mathrm{Na}$ & $2^{\text {nd }}$ solvation shell of $\mathrm{Na}^{+}$ \\
\hline $1 \mathrm{X} 2 \mathrm{X}$ & $1^{\text {st }}$ solvation shell of one $\mathrm{X}^{-} \& 2^{\text {nd }}$ of another \\
\hline $1 \mathrm{X} 2 \mathrm{Na}$ & $1^{\text {st }}$ solvation shell of an $\mathrm{X}^{-} \& 2^{\text {nd }}$ of an $\mathrm{Na}^{+}$ \\
\hline $1 \mathrm{Na} 1 \mathrm{X}^{a}$ & $1^{s t}$ solvation shell of an $\mathrm{Na}^{+} \& 1^{s t}$ of an $\mathrm{X}^{-}$ \\
\hline $1 \mathrm{Na} 2 \mathrm{Na}$ & $1^{\text {st }}$ solvation shell of one $\mathrm{Na}^{+} \& 2^{\text {nd }}$ of another \\
\hline $1 \mathrm{Na} 2 \mathrm{X}$ & $1^{\text {st }}$ solvation shell of an $\mathrm{Na}^{+} \& 2^{\text {nd }}$ of an $\mathrm{X}^{-}$ \\
\hline $2 \mathrm{Na} 2 \mathrm{X}$ & $2^{\text {nd }}$ solvation shell of an $\mathrm{Na}^{+} \& 2^{\text {nd }}$ of an $\mathrm{X}^{-}$ \\
\hline 3 solv & Solvation shells $\left(1^{\text {st }}\right.$ or $\left.2^{\text {nd }}\right)$ of three different ions \\
\hline Bulk & Outside the $1^{s t}$ or $2^{\text {nd }}$ solvation shell of any ion \\
\hline
\end{tabular}

approach is to consider an Arrhenius description, $D=A e^{-\beta E_{a}}$ and use the prefactor, $A$, as a proxy for the entropy-determined factors. A more explicit, but also approximate, approach is to consider a transition state theory-like description as

$$
D(T) \simeq D_{A} e^{-\beta \Delta A^{\ddagger}}=D_{A} e^{-\beta \Delta U^{\ddagger}} e^{\Delta S^{\ddagger} / R},
$$

where $D_{A}$ can be though of as an "attempt frequency" factor that has the units of $D$ and $\Delta A^{\ddagger}$, $\Delta U^{\ddagger}$, and $\Delta S^{\ddagger}$ are the activation (Helmholtz) free energy, internal energy, and entropy respectively. It is important to note that these quantities implicitly depend on the definition of a transition state dividing surface based on a reaction coordinate and are thus not explicitly measurable, in contrast to the activation energy which is determined by how $D$ changes with temperature.

With that caveat in mind, we can estimate the relative change in activation entropy from that of neat water with two assumptions: 1) $D_{A}$ is the same for waters in all environments, and 2) the activation internal energy can be approximated by the activation energy, $\Delta U^{\ddagger} \simeq$ $E_{a}$. Then, for a given population $\alpha$ of water molecules,

$$
\frac{D_{\alpha}}{D_{0}}=e^{-\beta\left(E_{a, \alpha}-E_{a, 0}\right)} e^{\left(\Delta S_{\alpha}^{\ddagger}-\Delta S_{0}^{\ddagger}\right) / R} .
$$

This relative measure of the activation entropy, $\Delta \Delta S_{\alpha}^{\ddagger}=\Delta S_{\alpha}^{\ddagger}-\Delta S_{0}^{\ddagger}$, is then given by

$$
\Delta \Delta S_{\alpha}^{\ddagger}=R \ln \left(\frac{D_{\alpha}}{D_{0}}\right)+\frac{E_{a, \alpha}-E_{a, 0}}{T} .
$$

This expression reflects the general expectation that differences in $D$ that are not associated with the activation energy are attributable to entropy. This neglects possible effects due to changes in the attempt frequency $D_{A}$ or transition state recrossing.

\section{Computational Details}

All MD simulations were performed using the Large-Scale Atomic/Molecular Massively Parallel Simulator (LAMMPS). $\$ 5960$ Each simulation consisted of 331 water molecules, described by the SPC/E model, $\stackrel{57}{5}$ and 6 ion pairs, using the Joung-Cheatham model. ${ }^{58}$ The force field parameters for each ion are shown in Table 2, Lorentz-Berthelot mixing rules were used. $\underline{6162}$ A total of 4 electrolyte solutions were consid- 


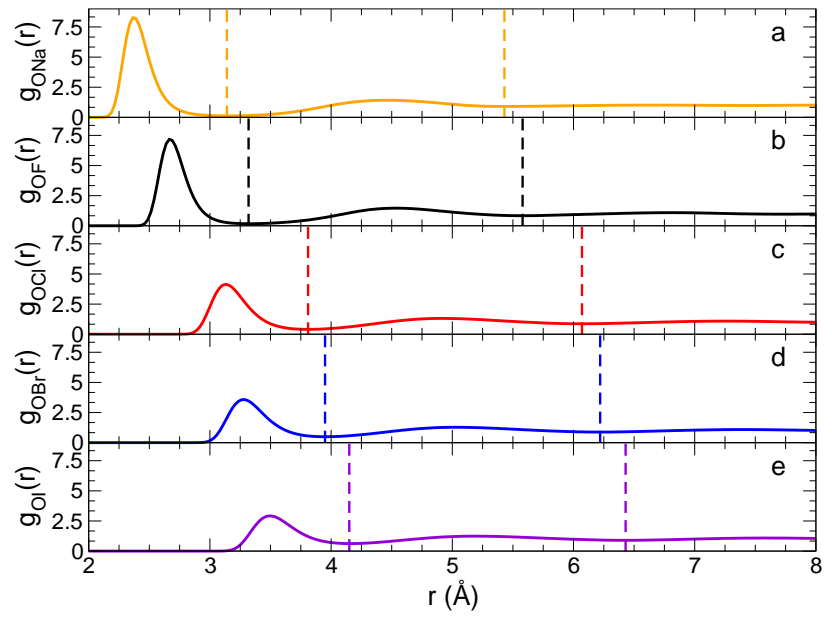

Figure 2: Water oxygen-ion radial distribution functions for $\sim 1 \mathrm{M}$ sodium-halide aqueous solutions. Results are shown where the ion is a) $\mathrm{Na}^{+}$(orange), b) $\mathrm{F}^{-}$(black), c) $\mathrm{Cl}^{-}$(red), d) $\mathrm{Br}^{-}$(blue), and e) $\mathrm{I}^{-}$(violet). Dashed vertical lines indicate the $1^{\text {st }}$ and $2^{\text {nd }}$ solvation shell cutoff distances for each case.

ered, all having $\mathrm{Na}^{+}$as the cation, and with the halides $\mathrm{F}^{-}, \mathrm{Cl}^{-}, \mathrm{Br}^{-}$, and $\mathrm{I}^{-}$as the anion. Each electrolyte system was first equilibrated for $500 \mathrm{ps}$ and then run for $10 \mathrm{~ns}$ at constant pressure and temperature using an $N p T$ ensemble with a Nosé-Hoover barostat with a pressure and temperature chain length of three. The damping parameters for the barostat and the thermostat were 1000 and 100 fs, respectively. The latter stage was used to determine the average volume, which was then used in subse-

Table 2: Force field parameters used in the simulations.

\begin{tabular}{cccc}
\hline Atom & $\sigma(\AA)$ & $\epsilon(\mathrm{kcal} / \mathrm{mol})$ & $q(e)$ \\
\hline \multicolumn{4}{c}{$\mathrm{SPC} / \mathrm{E} \mathrm{H}{ }_{2} \mathrm{O}$} \\
\hline $\mathrm{H}$ & 0.000 & 0.0000 & 0.4238 \\
$\mathrm{O}$ & 3.166 & 0.15535 & -0.8476 \\
\hline \multicolumn{4}{c}{ Joung-Cheatham Ions } \\
\hline $\mathrm{Na}^{+}$ & 2.160 & 0.3526418 & 1.000 \\
$\mathrm{~F}^{-}$ & 4.022 & 0.0074005 & -1.000 \\
$\mathrm{Cl}^{-}$ & 4.830 & 0.0127850 & -1.000 \\
$\mathrm{Br}^{-}$ & 4.902 & 0.0269586 & -1.000 \\
$\mathrm{I}^{-}$ & 5.201 & 0.0427845 & -1.000 \\
\hline
\end{tabular}

quent NVT ensemble simulations. The differences in average volume give rise to small deviations from the nominal $1 \mathrm{M}$ concentration; the precise values are given in Table S1.

Based on the calculated equilibrium volume, constant volume and temperature simulations were run for 1 ns of equilibration and 50 ns of production. A Nosé-Hoover thermostat (again with a chain length of three and a damping parameter of $100 \mathrm{fs}$ ) was used. During the latter, the configurations and momenta were written every 1 ps. These were used as the initial condition for 50,000 NVE trajectories of $20 \mathrm{ps}$ length from which the configurations were saved every 10 fs. These results were used to calculate the diffusion coefficients and activation energies; the approach removes any effect of the thermostat on the dynamics of interest.

In every simulation the timestep was 1 fs, the SHAKE algorithm was used for the water molecules, and the electrostatics were calculated using the particle-particle particle-mesh Ewald summation with an accuracy parameter of $1 \times 10^{-4}$. Previous studies have shown that the diffusion coefficient is sensitive to the size of the periodic boundary condition of the simulation box. ${ }^{63}$ Here we neglect this correction, which has been shown to not significantly affect the relative behavior of different anions $^{20}$ or the diffusion activation energy. Errors in computed quantities were obtained by block averaging using 20 blocks (each consists of 2,500 NVE trajectories) and are reported as $95 \%$ confidence intervals using the Student's $t$ distribution. 64

\section{Results and Discussion}

We now turn our attention to examining the diffusion coefficients and activation energies for water molecules in the sodium-halide aqueous solutions. We first examine the populations of water molecules in the categories (Table 1) defined by proximity to the ions. 


\section{Water Count}

In the analysis of the diffusion coefficients and activation energies presented below, a key component of understanding the effect of the ions is the classification of water molecules based on their location in the ion solvation shells. The percentage of water molecules in each of the categories listed in Table 1 are plotted in Fig. 3 for the four sodium-halide systems.

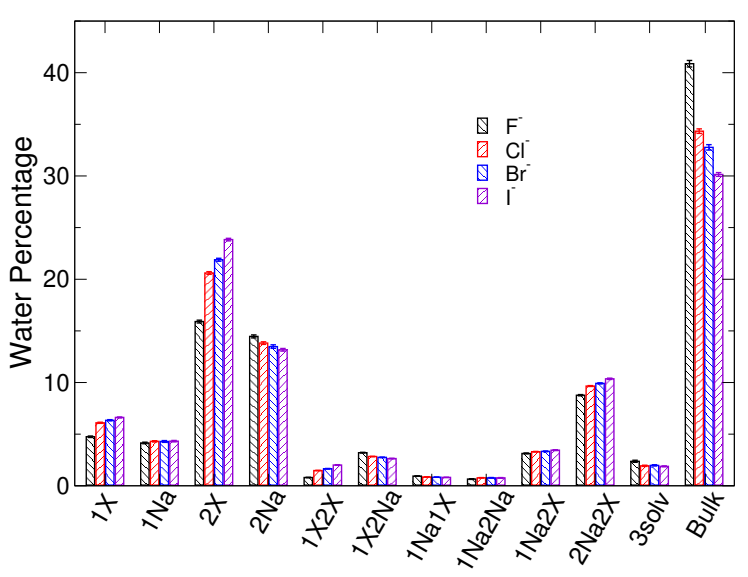

Figure 3: Average percentage of water molecules in each solvation shell category defined in Table 1 for $\sim 1 \mathrm{M}$ sodium-halide solutions.

We can first consider water molecules that are only within a $1^{\text {st }}$ solvation shell of one of a single ion. In the case of $\mathrm{Na}^{+}$, the percentage of water molecules that are only in the first solvation shell $(1 \mathrm{Na})$ is $\sim 4.3 \%$ and is essentially independent of the identity of the halide. This corresponds to $\sim 2.3 \mathrm{H}_{2} \mathrm{O}$ molecules per ion, which is not to be confused with the $\mathrm{Na}^{+}$coordination number $(\mathrm{CN})$ given from the sum of all categories involving $1 \mathrm{Na}$. We find $C N_{N a}=5.2$, consistent with previous simulation studies. $\frac{5165}{65}$

The percentage of water molecules that are only in the $1^{\text {st }}$ solvation shell of a halide is greater and increases moving down the group from 4.7 to $6.6 \%$. Namely, we find 15.7, 20.2, 21.0, and $21.9 \mathrm{H}_{2} \mathrm{O}$ molecules in the $1 \mathrm{~F}, 1 \mathrm{Cl}$, $1 \mathrm{Br}$, and $1 \mathrm{I}$ categories, corresponding to 2.6 , 3.4, 3.5, and 3.7 molecules per $\mathrm{F}^{-}, \mathrm{Cl}^{-}, \mathrm{Br}^{-}$, and $\mathrm{I}^{-}$ion, respectively. Adding the results for all categories in which a water molecule is in the $1^{s t}$ solvation shell of an anion gives the coordi- nation numbers for the halides as $C N_{F}=5.3$, $C N_{C l}=6.2, C N_{B r}=6.4, C N_{I}=6.7$, which are also in agreement with prior work. $\frac{45166] 69}{616}$

There are significantly more water molecules that are solely in the $2^{\text {nd }}$ solvation shell of an ion due to the larger volumes involved. The numbers in this category increase for the $2 \mathrm{X}$ solvation shells moving down the halide series from $52.6 \mathrm{H}_{2} \mathrm{O}$ for $2 \mathrm{~F}$ to 78.9 molecules for $2 \mathrm{I}$ (between 15.9 to $23.9 \%$ of all water molecules respectively). However, they decrease (albeit quite weakly) for the $2 \mathrm{Na}$ category for the same series from $47.9\left(\mathrm{~F}^{-}\right)$to $43.6\left(\mathrm{I}^{-}\right)$(between 14.5 to $13.2 \%$ of all water molecules, respectively).

There are five classifications of the water molecules in multiple solvation shells that occur with only a modest water population ( $\leq$ $3 \%$ water molecules): 1X2X, 1X2Na, $1 \mathrm{X} 1 \mathrm{Na}$, $1 \mathrm{Na} 2 \mathrm{Na}$, and 3solv. The total for all these categories is between 26.1 and $26.8 \mathrm{H}_{2} \mathrm{O}$ molecules for the different anion cases. As will be seen below, the limited number of molecules in some of these categories leads to larger error bars in the dynamical properties. The small populations are a consequence of the concentration; these environments will have a more significant impact on the overall behavior of more concentrated electrolyte solutions.

Two multiple solvation shell classifications of water molecules occur with larger populations: $1 \mathrm{Na} 2 \mathrm{X}$ and $2 \mathrm{Na} 2 \mathrm{X}$. Both exhibit modest increases in the number of $\mathrm{H}_{2} \mathrm{O}$ molecules moving down the halide series. The number in the $1 \mathrm{Na} 2 \mathrm{X}$ category increases from 10.4 to 11.4 and in the $2 \mathrm{Na} 2 \mathrm{X}$ from 29.0 to 34.3 from $\mathrm{F}^{-}$to $\mathrm{I}^{-}$.

Finally, the largest category of water molecules in these $\sim 1 \mathrm{M}$ simulations is outside the $1^{\text {st }}$ or $2^{\text {nd }}$ solvation shell of any ion, i.e., "Bulk." The number of Bulk $\mathrm{H}_{2} \mathrm{O}$ molecules decreases with the size of the ion because, as we noted above, more waters are part of the halide solvation shells moving down the halide group. Thus, we find that an average of 135.3 Bulk water molecules for the $\mathrm{NaF}$ solution, corresponding to $40.9 \%$ of all the waters in the solution. This number is significantly reduced even for the $\mathrm{NaCl}$ solution to $113.6(34.3 \%)$ and it becomes slightly smaller for the $\mathrm{NaBr}(108.5$ molecules; 32.8\%) and NaI (99.8 molecules; 

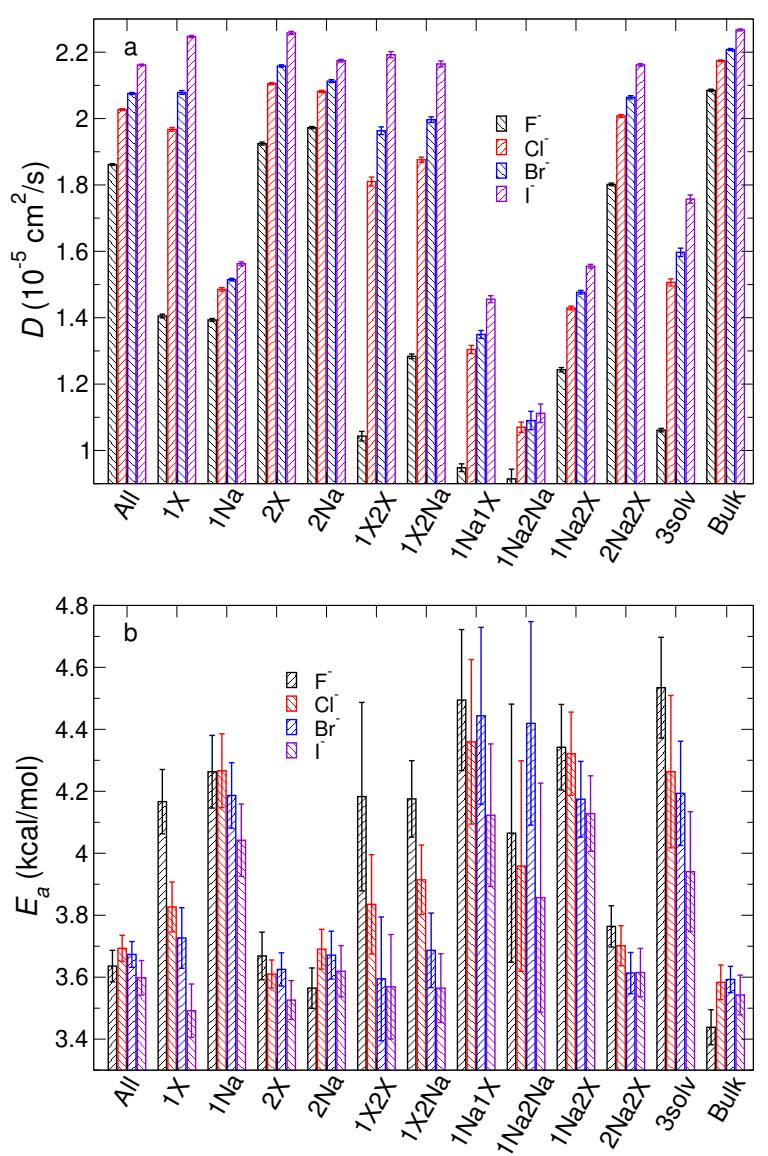

Figure 4: a) Diffusion coefficients and b) corresponding activation energies for water molecules in different initial locations relative to ions in $\sim 1 \mathrm{M}$ sodium halide solutions.

$30.1 \%$ ). As will be seen below, water molecules in this Bulk classification should not be thought of as identical to those in neat water, but rather only as water molecules in the solution that do not fall within the first two solvation shells of any ion.

\section{Diffusion Coefficients}

The water diffusion coefficients in the four $\sim$ $1 \mathrm{M}$ sodium-halide solutions were calculated and subdivided based on the initial location of the water oxygen atom based on the categories defined in Table 1 and illustrated in Figs. 1 and 2. The results are presented in Fig. 4 a and the values are provided in Table S3 and plotted separately for each category in Figs. S3 and S5-S7. For every category, the diffusion coefficient is slowest for $\mathrm{F}^{-}$and increases moving down the period to $\mathrm{I}^{-}$. This is consistent with what has been observed previously in numerous experiments $^{1 / 20|24| 25 \mid 27}$ and simulations s0|32|35|36/70|71 $_{\text {of }}$ alkali-halide solutions. The magnitude of the changes in $D$ with the identity of the halide, however, depends strongly on the initial location of the water molecule, which is examined below.

Quantitatively, we obtain diffusion coefficients of $1.861 \pm 0.003,2.027 \pm 0.002,2.076 \pm$ 0.003 , and $2.161 \pm 0.003$ in units of $10^{-5} \mathrm{~cm}^{2} / \mathrm{s}$ when averaging over all the water molecules in the $\mathrm{NaF}, \mathrm{NaCl}, \mathrm{NaBr}$, and $\mathrm{NaI}$ solutions, respectively. All are lower than the $D_{0}=$ $(2.444 \pm 0.003) \times 10^{-5} \mathrm{~cm}^{2} / \mathrm{s}$ obtained for neat water. Compared to NMR measurements, the simulation model systematically overestimates the slowdown due to the ions, but captures the trend (even quantitatively). For $\mathrm{NaCl}, \mathrm{NaBr}$, and $\mathrm{NaI}$ our results give $D / D_{0}=0.83,0.85$, and 0.88 , respectively, while reported measured values are $\sim 0.93,1124 \sim 0.94-0.96, \stackrel{24 \mid 27}{,}$ and $\sim 0.97-0.98, \frac{124 \mid 27}{12}$ respectively.

The behavior of the water diffusion coefficients shown in Fig. $4 \mathrm{a}$ is consistent with the ranking of the halides within the Hofmeister series, in which $\mathrm{F}^{-}$is the strongest kosmotrope and $\mathrm{I}^{-}$is the most chaotropic. It is interesting that this behavior is independent of the local environment of the water molecule and persists even for water molecules that are in the first solvation shell of $\mathrm{Na}^{+}$and outside the first two solvation shells of an anion.

The effect of $\mathrm{Na}^{+}$on the water molecule diffusion is greater than that of any of the halides, but comparable to $\mathrm{F}^{-}$. This is apparent in the $1 \mathrm{X}$ and $1 \mathrm{Na}$ diffusion coefficients, where the latter are smaller than the former for all halides except $\mathrm{F}^{-}$where they are the same within error. This is suggestive of a size, or more particularly a charge density effect, given that $\mathrm{Na}^{+}$and $\mathrm{F}^{-}$are isoelectronic while the other halides are significantly larger. Similar behavior is found in comparing within the $2 \mathrm{~F}$ and $2 \mathrm{Na}$ and the $1 \mathrm{~F} 2 \mathrm{Na}$ and $1 \mathrm{Na} 2 \mathrm{~F}$ pairs of categories. In the case of the former, $D$ is slightly $(\sim 3 \%)$ smaller for water molecules in the $2^{\text {nd }}$ solvation shell of $\mathrm{F}^{-}$than for $\mathrm{Na}^{+}$, but the reverse is true for water molecules in shared solvation shells, where 
$D(1 \mathrm{~F} 2 \mathrm{Na})$ is larger than $D(1 \mathrm{Na} 2 \mathrm{~F})$ by roughly the same factor.

The slowest diffusion is observed for water molecules that are in the $1^{\text {st }}$ solvation shell of one $\mathrm{Na}^{+}$and the $2^{\text {nd }}$ solvation shell of another $(1 \mathrm{Na} 2 \mathrm{Na})$ and in the solvent-separated ion pair of an $\mathrm{Na}^{+}$and an $\mathrm{F}^{-}(1 \mathrm{~F} 1 \mathrm{Na})$. These water molecules have diffusion coefficients $\sim 2.2$ times smaller than that of the Bulk water in the $\mathrm{NaF}$ solution. These results indicate the significant effect of the sodium cation.

\section{Activation Energy of Diffusion}

The total activation energy of diffusion, $E_{a}$, was calculated from the four $\sim 1 \mathrm{M}$ sodium-halide solutions using their respective diffusion coefficients for each water category and the results are presented in Fig. 4 $4 \mathrm{~b}$; the values are provided in Table S4 and plotted separately in Figs. S2, $\mathrm{S} 4, \mathrm{~S} 6$, and S7. One expects that, if the trends in $D$ with halide shown in Fig. 伴 are due to energetic effects, the activation energy should decrease moving down the halide group. This expectation is borne out for many of the water molecule populations, particularly 1X, 1X2X, $1 \mathrm{X} 2 \mathrm{Na}, 1 \mathrm{Na} 2 \mathrm{X}, 2 \mathrm{Na} 2 \mathrm{X}$, and 3solv. It also appears to be the case for some of the other categories $(1 \mathrm{Na}, 2 \mathrm{X}$, and $1 \mathrm{Na} 1 \mathrm{X})$ where the trend is not as easily discerned.

Interestingly, three remaining water populations - All, 2Na, and Bulk - suggest a different trend that is not consistent with a purely energetic (as opposed to entropic) explanation for the halide dependence of $D$. (The $1 \mathrm{Na} 2 \mathrm{Na}$ case involves too few molecules and does not show a clear trend.) Namely, we find $E_{a}$ is smaller for the $\mathrm{F}^{-}$and $\mathrm{I}^{-}$cases and higher for $\mathrm{Cl}^{-}$and $\mathrm{Br}^{-}$, which are nearly equivalent. The halide dependence in these populations is weak, but clearly not consistent with $\mathrm{F}^{-}$giving rise to the largest activation energy.

Most importantly, this includes the experimentally accessible All case where all waters are included in the average, where we obtain $E_{a, A l l}=3.64 \pm 0.05,3.70 \pm 0.04,3.67 \pm 0.04$, and $3.60 \pm 0.06 \mathrm{kcal} / \mathrm{mol}$ for $\mathrm{NaF}, \mathrm{NaCl}, \mathrm{NaBr}$, and NaI, respectively. These values are the same as, or nearly so, that calculated for neat water,
$3.60 \pm 0.04 \mathrm{kcal} / \mathrm{mol}$. This is generally consistent with the limited measured results that have been reported, e.g., that indicate the water diffusion coefficient activation energy of $1 \mathrm{~mol} / \mathrm{kg}$ $\mathrm{NaCl}$ is nearly the same as that for neat water, $\frac{33}{3}$ as well as the Arrhenius analysis of MD results by Kim et al. ${ }^{[20}$ The trend for the All category is at least partly attributable to the large fraction of the total number of water molecules in the Bulk and 2Na categories (Fig. 3). These results suggest that the measurable trend in $E_{a}$ with halide for all water molecules in the solution will change with increasing salt concentration as the different populations change and, particularly, the number of Bulk molecules declines.

Note the $E_{a}$ is consistently large within the $1 \mathrm{Na}$ category compared to the $1 \mathrm{X}$ category. This larger trend in $E_{a}$ is also apparent in overlapping solvation shells that include the $1 \mathrm{Na}$ category: $1 \mathrm{Na} 1 \mathrm{X}, 1 \mathrm{Na} 2 \mathrm{Na}$, and $1 \mathrm{Na} 2 \mathrm{X}$. Some previous studies reported in the literature have argued that the cation has a smaller effect than the anion on water structure ${ }^{18}$ and dynamics. ${ }^{21}$ For example, Gaiduk and Galli found that, in density functional theory-based MD simulations of $0.87 \mathrm{M} \mathrm{NaCl}^{-\mathrm{Cl}^{-}}$affected the average number of $\mathrm{H}$-bonds per water molecule out to the $3^{\text {rd }}$ solvation shell while $\mathrm{Na}^{+}$only had an effect on the $1^{\text {st }}$ solvation shell.

\section{Mechanism of Anion Effects in the $1^{\text {st }}$ Solvation Shell}

It is interesting to consider in greater detail the simplest ion effects by examining the cases where a water molecule is immediately adjacent to an ion. We first consider the effect of the anion by examination of the $1 \mathrm{X}$ population dynamics. The diffusion $E_{a}$ is significantly enhanced, compared to the neat water value of $3.60 \pm 0.04 \mathrm{kcal} / \mathrm{mol}$, for water molecules in the $1^{\text {st }}$ solvation shell of an $\mathrm{F}^{-}(4.17 \pm$ $0.10 \mathrm{kcal} / \mathrm{mol}$ ) and, to a lesser degree, $\mathrm{Cl}^{-}$ $(3.83 \pm 0.08 \mathrm{kcal} / \mathrm{mol})$. On the other hand, the effect is much smaller for $\mathrm{Br}^{-}$and $\mathrm{I}^{-}$for which $E_{a, 1 X}=3.73 \pm 0.10$ and $3.49 \pm 0.09 \mathrm{kcal} / \mathrm{mol}$, respectively.

We can explore the origin of these changes 


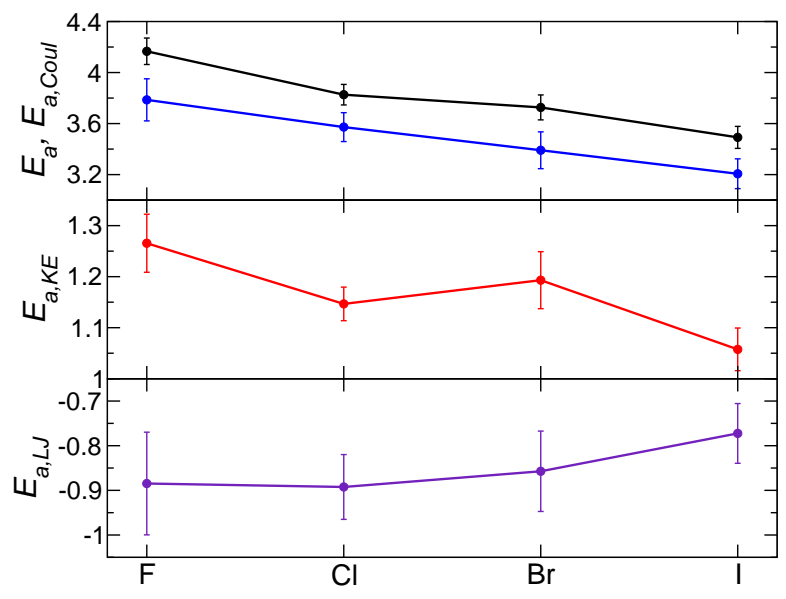

Figure 5: Decomposition of the water diffusion $E_{a}$ (black) for molecules in the $1^{\text {st }}$ solvation shell of a halide (1X); Coulombic (blue), kinetic (red) and Lennard-Jones (violet) energy components (all in $\mathrm{kcal} / \mathrm{mol}$ ) are shown.

using the decomposition of $E_{a}$ described in the "Theory" section, the results for which are shown in Fig. 5. As we have previously observed for neat water, $47 / 49$ electrostatic interactions are the dominant contribution to the $E_{a}$ and nearly equal the total activation energy. The Coulombic component is partially cancelled by the Lennard-Jones term, which has a clear physical interpretation. The water donating an $\mathrm{H}$-bond to the halide anion sits up on the repulsive wall of the Lennard-Jones potential; note that the $g_{O X}(r) 1^{\text {st }}$ solvation shell peak (Fig. 2) occurs at distances less than $\left(\sigma_{O}+\sigma_{X}\right) / 2$ (Table 2). This occurs because it permits favorable Coulombic interactions between the water $\mathrm{H}$ and the anion. Thus, for a water molecule to diffuse, which must of necessity involve the breakage of this water-anion H-bond, it must move up the Coulombic potential while it simultaneously falls down to lower Lennard-Jones energies. This is the origin of the strong positive values of $E_{a, \text { Coul }}$ and the negative values of $E_{a, L J}$. The former involve the dominant interactions and are thus larger in magnitude.

The results in Fig. 5 show that this competition between the Coulombic and Lennard-Jones contributions differs quantitatively as we move down the halide group. Namely, the water-

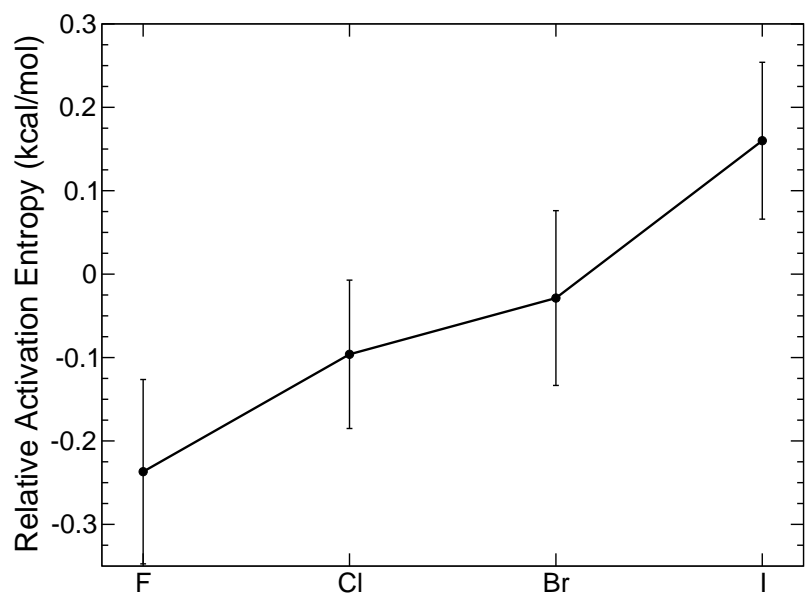

Figure 6: Relative activation entropy contribution for diffusion, $-T \Delta \Delta S^{\ddagger}$ from eq. 13 , for water molecules in the $1^{\text {st }}$ solvation shell of a halide (1X).

halide electrostatic interactions become weaker as the size of the anion increases. This leads to the water molecule sitting lower down on the Lennard-Jones repulsive wall so that $E_{a, L J}$ increases (becomes less negative). Overall, the leads to a decline in $E_{a}$ for larger halides. (There is an additional small contribution to this trend from changes in $E_{a, K E}$ for which we do not currently have a simple physical interpretation.)

It is important to note, however, that the activation energies, with the underlying competition between interactions, are not sufficient to describe the change in $D$ for water molecules in the halide $1^{\text {st }}$ solvation shell. This can be seen by calculating the activation entropy, relative to neat water, through eq. 13 , the results for which are shown in Fig. 6. The data indicates that entropic effects represent a significant contribution to the trend in $D$ along the halide series. Specifically, the diffusion activation entropy of water molecules adjacent to an $\mathrm{F}^{-}$or $\mathrm{Cl}^{-}$ion is greater than that in neat water; this entropic effect would tend to make $D$ larger, but the larger $E_{a, 1 X}$ values dominate, leading to slower diffusion. These contributions are reversed for $\mathrm{I}^{-}$where $\Delta \Delta S^{\ddagger}<0$ but $E_{a, 1 I}<E_{a, 0}$ and the former leads to a smaller $D$ than for neat water. In the case of water molecules in the $1^{\text {st }}$ solvation shell of $\mathrm{Br}^{-}$, the slower diffusion can be 


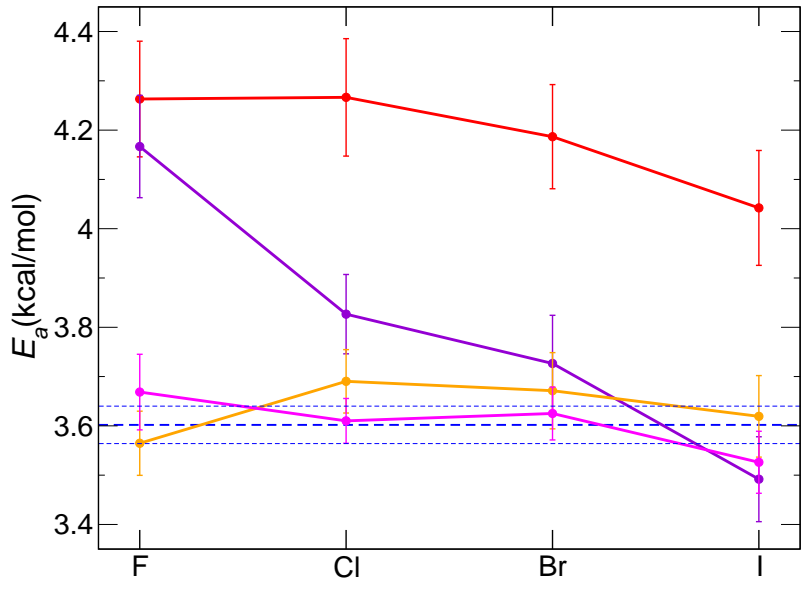

Figure 7: Diffusion activation energy, $E_{a}$, for water molecules in the $1 \mathrm{Na}$ (red), $1 \mathrm{X}$ (violet), $2 \mathrm{Na}$ (orange), and 2X (magenta) populations; the neat water result is shown for comparison (dashed blue line with uncertainties indicated by lighter dashed lines).

nearly completely accounted for by a slightly larger activation energy and $\Delta \Delta S^{\ddagger} \approx 0$. (At this juncture, it is important to recall the assumptions used to obtain eq. 13, which make the trends in $\Delta \Delta S^{\ddagger}$ more meaningful than the quantitative values.)

\section{Length Scale of Ion Effects}

It is useful to examine the spatial extent of the effect of the ions on the water diffusion activation energies. Such a comparison is made in Fig. 7, where $E_{a}$ is plotted for water molecules that fall within the $1^{\text {st }}$ or $2^{\text {nd }}$ solvation shell of either $\mathrm{Na}^{+}$or a halide ion, i.e., the $1 \mathrm{X}, 1 \mathrm{Na}$, $2 \mathrm{X}$, and $2 \mathrm{Na}$ populations. The activation energy is largest for waters within the $1^{\text {st }}$ solvation shell of $\mathrm{Na}^{+}$followed by waters within the $1^{\text {st }}$ solvation shells of the halide ions. Both $E_{a, 1 N a}$ and $E_{a, 1 X}$ decrease moving down the series of halides, with the former exhibiting relatively modest changes while the latter drops by more than $0.6 \mathrm{kcal} / \mathrm{mol}$. Note that the $E_{a, 1 N a}$ is heavily dominated by the Coulombic contribution (see Fig. S2) as was seen in the previous section for $1 \mathrm{X}$.

However, for water molecules in the $2^{\text {nd }}$ solvation shell of an ion, the activation energies are

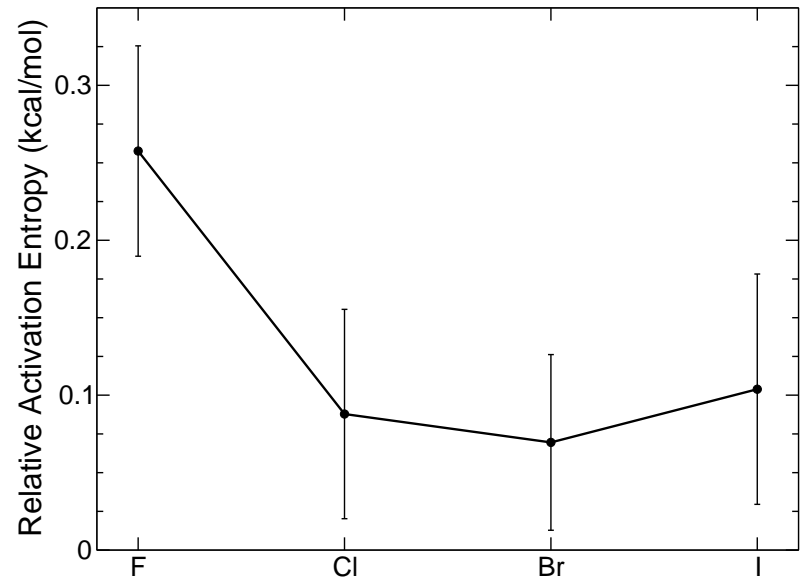

Figure 8: Relative activation entropy contribution for diffusion, $-T \Delta \Delta S^{\ddagger}$ from eq. 13 , for water molecules outside any ion $1^{\text {st }}$ or $2^{\text {nd }}$ solvation shell (Bulk).

the same, within error, as that for neat water. The data for these $2 \mathrm{Na}$ and $2 \mathrm{X}$ categories shown in Fig. 7 hint at some changes with the identity of the halide but no differences can be resolved outside of the statistical uncertainties. This suggests that, at the activation energy level, the effect of individual sodium and halide ions on water diffusion is primarily restricted to the first solvation shell. It is clear from Fig. 4b that water molecules in overlapping solvation shells manifest much stronger effects of interactions with ions in their diffusion activation energy.

In this context, it is interesting to compare the results for the Bulk water category to that for neat water. From the results in Fig. 4b, it is clear that $E_{a, B u l k}$ is effectively the same as that, $3.60 \pm 0.04 \mathrm{kcal} / \mathrm{mol}$, obtained in our simulation of neat water for $\mathrm{Cl}^{-}, \mathrm{Br}^{-}$, and $\mathrm{I}^{-}$, but it is distinctly lower for the NaF solution. These values are suggestive of significant entropic effects on water diffusion, given that $D_{B u l k}$ is less than the neat water value for all of the ionic solutions considered here. Moreover, $D_{\text {Bulk }}$ is lowest for the $\mathrm{NaF}$ case for which the activation energy is also lowest. The suggests a longer-range effect for the entropic factors governing diffusion compared to that for the energetic driving forces (here measured by the activation energy).

These entropic effects can be estimated by the relative activation entropy, $\Delta \Delta S^{\ddagger}$ in eq. 13 , 
which is plotted for Bulk water molecules in Fig. 8 as a function of halide. The results presented in the figure indicate that the activation entropy of "Bulk" water in all of the salt solutions is smaller than that for neat water. For the larger halides (chloride through iodide) this entropic factor accounts for the slower diffusion of uncoordinated waters in the salt solutions given the equality of the activation energies. A significantly larger entropic effect is observed for the $\mathrm{NaF}$ solution, which more than compensates for its lower activation energy, relative to neat water or Bulk water in the other sodium halide solutions. The nature and origin of these entropic effects that extend well outside the first two ion solvation shells is still unclear and a more direct investigation of this behavior is needed.

\section{Conclusions}

We have utilized a method of dividing up water populations in sodium halide solutions to gain insight into how water behaves in the proximity of an ion as a function of the identity of and distance from the ion. For each water population so defined, the diffusion coefficient, $D$, and activation energy of diffusion, $E_{a}$, have been calculated. The activation energies are obtained using a fluctuation theory for dynamics approach that gives $E_{a}$ from single temperature MD simulations. $\stackrel{46}{49}$ It further provides mechanistic insight by decomposition of the activation energy into components from the interactions and motions present in the system. This work has yielded several interesting observations.

First, the diffusion coefficient of water in sodium-halide solutions follows a trend anticipated by the Hofmeister series and observed in previous measurements and simulations. Namely, $D$ increases moving down the halide group from fluoride to iodide. This is true not only when averaging over all the water molecules in the solution, but also for each population of water molecules.

Second, the sodium ion exhibits a stronger effect on water diffusion than any of the halides, though the behavior of $\mathrm{F}^{-}$is nearly comparable.
Third, the Hofmeister series is not reflected in the same way in the diffusion coefficient activation energy. While several water molecule environments (categorized by the proximity to an ion or ions) do follow the trend expected from the Hofmeister series for the halides, the quantitative changes in $E_{a}$ are not sufficient to explain the behavior of the diffusion coefficient. In addition, the $E_{a}$ for some populations of water molecules do not follow predictions based on the Hofmeister series.

Fourth, this behavior of $D$ and $E_{a}$ point strongly to an important role for entropic effects that are ion dependent. Because the activation entropy cannot be unambiguously calculated, these factors are harder to quantify; we have estimated them from a transition state theory-like approximation. As we generally observe energetic-entropic compensation, the estimated $\Delta \Delta S^{\ddagger}$, like the activation energies, differ between the various solvation shell-based water molecule populations. A key observation is that, for water molecules in the first solvation shell of a halide, entropic effects increasingly disfavor water diffusion with larger halide size.

Fifth, the decomposition of the activation energy into contributions from the kinetic, Lennard-Jones, and Coulombic energies indicates a strong competition between the latter two, as has been observed previously in neat water. This competition plays out most clearly for water molecules that are in the first solvation shell of (donating an H-bond to) a halide ion. The larger the anion the weaker the Coulombic attraction in the $\mathrm{H}$-bond and the lower on the Lennard-Jones repulsive wall the H-bonding water molecule sits. This leads to a decreasing Coulombic contribution to $E_{a}$ moving from $\mathrm{F}^{-}$to $\mathrm{I}^{-}$that is partially compensated by a smaller Lennard-Jones component that increases (grows less negative).

It will be interesting to use analogous activation energy calculations to explore several other aspects of ion effects on water dynamics. Examination of the trends in water diffusion with alkali metal cation in comparison to Hofmeister series predictions is of interest. Similarly, the effect of salt concentration has been found to exhibit interesting (sometimes 
non-intuitive (3013133 $^{(3 f f e c t s}$ on water diffusion. The relative role of energetic and entropic contributions to diffusion as well as electrostatic and Lennard-Jones interaction components of the activation energy in water diffusion around polyatomic and multiply-charged ions requires further investigation. Finally, it is of interest to explore how other water dynamical processes - e.g., OH reorientation, viscosity, and vibrational spectroscopy - might exhibit different temperature dependences than diffusion.

\section{Supporting Information}

The Supporting Information is available free of charge on the ACS Publications website at DOI:

Additional simulation details, $\mathrm{O} \cdots \mathrm{Na}$ radial distribution functions, solvation shell cutoff distances, numerical values and additional plots of diffusion coefficients and activation energies.

\section{Acknowledgments}

This work was supported by the National Science Foundation under Grant No. CHE1800559. A.K.B. gratefully acknowledges support from a University of Kansas Dean's Doctoral Fellowship. This material is based upon work supported by the National Science Foundation Graduate Research Fellowship under Grant Nos. 1540502 and 1451148 (Z.A.P.). The calculations were performed at the University of Kansas Center for Research Computing (CRC).

\section{References}

(1) Harris, K. R.; Mills, R.; Back, P. J.; Webster, D. S. An improved NMR spin-echo apparatus for the measurement of selfdiffusion coefficients: The diffusion of water in aqueous electrolyte solutions. Biophys. J. 1978, 29, 473-482.

(2) Collins, K. D.; Washabaugh, M. W. The Hofmeister effect and the behaviour of water at interfaces. Q. Rev. Biophys. 1985, 18, 323-422.
(3) Ohtaki, H.; Radnai, T. Structure and dynamics of hydrated ions. Chem. Rev. 1993, 93, 1157-1204.

(4) Bakker, H. J. Structural dynamics of aqueous salt solutions. Chem. Rev. 2008, 108, 1456-1473.

(5) Marcus, Y. Effect of ions on the structure of water: Structure making and breaking. Chem. Rev. 2009, 109, 1346-1370.

(6) Zangi, R. Can salting-in/salting-out ions be classified as chaotropes/kosmotropes? J. Phys. Chem. B 2010, 114, 643-650.

(7) Assaf, K. I.; Nau, W. M. The chaotropic effect as an assembly motif in chemistry. Angew. Chem. Int. Ed. 2018, 57, 1396813981.

(8) Oncsik, T.; Trefalt, G.; Borkovec, M.; Szilagyi, I. Specific ion effects on particle aggregation induced by monovalent salts within the Hofmeister series. Langmuir 2015, 31, 3799-3807.

(9) Okur, H. I.; Hladílková, J.; Rembert, K. B.; Cho, Y.; Heyda, J.; Dzubiella, J.; Cremer, P. S.; Jungwirth, P. Beyond the Hofmeister series: Ion-specific effects on proteins and their biological functions. J. Phys. Chem. B 2017, 121, 19972014 .

(10) Freire, M. G.; Neves, C. M.; Silva, A. M.; Santos, L. M.; Marrucho, I. M.; Rebelo, L. P.; Shah, J. K.; Maginn, E. J.; Coutinho, J. A. ${ }^{1} \mathrm{H}$ NMR and molecular dynamics evidence for an unexpected interaction on the origin of saltingin/salting-out phenomena. J. Phys. Chem. B 2010, 114, 2004-2014.

(11) Moilanen, D. E.; Wong, D. B.; Rosenfeld, D. E.; Fenn, E. E.; Fayer, M. D. IonWater hydrogen-bond switching observed with 2D IR vibrational echo chemical exchange spectroscopy. Proc. Natl. Acad. Sci. USA 2009, 106, 375-380. 
(12) Post, S. T. D.; Bakker, H. J. The combined effect of cations and anions on the dynamics of water. Phys. Chem. Chem. Phys. 2012, 14, 6280-6288.

(13) Giammanco, C. H.; Wong, D. B.; Fayer, M. D. Water dynamics in divalent and monovalent concentrated salt solutions. J. Phys. Chem. B 2012, 116, 13781-13792.

(14) Tielrooij, K. J.; Garcia-Araez, N.; Bonn, M.; Bakker, H. J. Cooperativity in ion hydration. Science 2010, 328, 1006-1009.

(15) Xie, W. J.; Gao, Y. Q. A simple theory for the Hofmeister series. J. Phys. Chem. Lett. 2013, 4, 4247-4252.

(16) Stirnemann, G.; Wernersson, E.; Jungwirth, P.; Laage, D. Mechanisms of acceleration and retardation of water dynamics by ions. J. Am. Chem. Soc. 2013, 135, 11824-11831.

(17) Stirnemann, G.; Jungwirth, P.; Laage, D. Water dynamics in concentrated electrolytes: Local ion effect on hydrogenbond jumps rather than collective coupling to ion clusters. Proc. Natl. Acad. Sci. USA 2018, 115, E4953-E4954.

(18) Gaiduk, A. P.; Galli, G. Local and global effects of dissolved sodium chloride on the structure of water. J. Phys. Chem. Lett. 2017, 8, 1496-1502.

(19) Xie, W. J.; Yang, Y. I.; Gao, Y. Q. Dual reorientation relaxation routes of water molecules in oxyanion's hydration shell: A molecular geometry perspective. J. Chem. Phys. 2015, 143, 224504.

(20) Kim, J. S.; Wu, Z.; Morrow, A. R.; Yethiraj, A.; Yethiraj, A. Self-diffusion and viscosity in electrolyte solutions. J. Phys. Chem. B 2012, 116, 12007-12013.

(21) Cremer, P. S.; Flood, A. H.; Gibb, B. C.; Mobley, D. L. Collaborative routes to clarifying the murky waters of aqueous supramolecular chemistry. Nat. Chem. 2018, 10, 8-16.

(22) Conway, B. E. Electrolyte solutions: Solvation and structural aspects. Annu. Rev. Phys. Chem. 1966, 17, 481-528.

(23) Hertz, H. G.; Holz, M.; Mills, R. The effect of structure on ion self-diffusion in concentrated electrolyte solutions. J. Chimie Phys. 1974, 71, 1355-1362.

(24) McCall, D. W.; Douglass, D. C. The effect of ions on the self-diffusion of water. I. Concentration dependence. J. Phys. Chem. 1965, 69, 2001-2011.

(25) Endom, L.; Hertz, H. G.; Thül, B.; Zeidler, M. D. A microdynamic model of electrolyte solutions as derived from nuclear magnetic relaxation and selfdiffusion data. Berich. Bunsen. Gesell. 1967, 71, 1008-1031.

(26) Engel, G.; Hertz, H. G. On the negative hydration. A nuclear magnetic relaxation study. Ber. Bunsen. Phys. Chem. 1968, 72, 808-834.

(27) Hertz, H. G.; Stalidis, G.; Versmold, H. The structure of concentrated electrolyte solutions as studied by nuclear magnetic resonance methods. J. Chimie Phys. 1969, 66, 177-188.

(28) Hertz, H. G.; Mills, R. The effect of structure on self-diffusion in concentrated electrolytes: Relationship between the water and ionic self-diffusion coefficients. $J$. Chimie Phys. 1976, 73, 499-508.

(29) Reiners, G.; Lorenz, W. J.; Hertz, H. G. Tracer diffusion-coefficients in aqueouselectrolyte solutions of various structure forming and breaking ions. Ber. Bunsen. Phys. Chem. 1978, 82, 738-744.

(30) Kim, J. S.; Yethiraj, A. A diffusive anomaly of water in aqueous sodium chloride solutions at low temperatures. $J$. Phys. Chem. B 2008, 112, 1729-1735. 
(31) Longinotti, M. P.; Carignano, M. A.; Szleifer, I.; Corti, H. R. Anomalies in supercooled $\mathrm{NaCl}$ aqueous solutions: A microscopic perspective. J. Chem. Phys. 2011, 134, 244510.

(32) Ding, Y.; Hassanali, A. A.; Parrinello, M. Anomalous water diffusion in salt solutions. Prod. Natl. Acad. Sci. USA 2014, 111, 3310-3315.

(33) Garbacz, P.; Price, W. S. ${ }^{1} \mathrm{H}$ NMR diffusion studies of water self-diffusion in supercooled aqueous sodium chloride solutions. J. Phys. Chem. A 2014, 118, 33073312 .

(34) Hewish, N. A.; Enderby, J. E.; Howells, W. S. The dynamics of water molecules in ionic solution. J. Phys. C: Solid State Phys. 1983, 16, 1777-1791.

(35) Joung, I. S.; Cheatham III, T. E. Molecular dynamics simulations of the dynamic and energetic properties of alkali and halide ions using water-model-specific ion parameters. J. Phys. Chem. B 2009, 113, 13279-13290.

(36) Kann, Z. R.; Skinner, J. L. A scaled-ioniccharge simulation model that reproduces enhanced and suppressed water diffusion in aqueous salt solutions. J. Chem. Phys. 2014, 141.

(37) Balbuena, P. B.; Johnston, K. P.; Rossky, P. J.; Hyun, J.-K. Aqueous ion transport properties and water reorientation dynamics from ambient to supercritical conditions. J. Phys. Chem. B 1998, 102, 3806-3814.

(38) Holzmann, J.; Ludwig, R.; Geiger, A.; Paschek, D. Pressure and salt effects in simulated water: Two sides of the same coin? Angew. Chem. Int. Edit. 2007, 46, 8907-8911.

(39) Woolf, L. A. Tracer diffusion of tritiated water (THO) in ordinary water $\left(\mathrm{H}_{2} \mathrm{O}\right)$ under pressure. J. Chem. Soc., Faraday Trans. 1 1975, 71, 784-796.
(40) Angell, C. A.; Finch, E. D.; Woolf, L. A.; Bach, P. Spin-echo diffusion coefficients of water to 2380 Bar and $-20^{\circ} \mathrm{C}$. J. Chem. Phys. 1976, 65, 3063-3074.

(41) Krynicki, K.; Green, C. D.; Sawyer, D. W. Pressure and temperature dependence of self-diffusion in water. Faraday Discuss. Chem. Soc. 1978, 66, 199-208.

(42) Harris, K. R.; Woolf, L. A. Pressure and temperature dependence of the self diffusion coefficient of water and oxygen-18 water. J. Chem. Soc., Faraday Trans. 1 1980, 76, 377-379.

(43) Weingartner, H. Self-diffusion in liquid water. A reassessment. Z. Phys. Chem. 1982, 132, 129-149.

(44) Harris, K. R.; Newitt, P. J. Self-diffusion of water at low temperatures and high pressure. J. Chem. Eng. Data 1997, 42, 346-348.

(45) Laage, D.; Stirnemann, G. Effect of ions on water dynamics in dilute and concentrated aqueous salt solutions. J. Phys. Chem. B 2019, 123, 3312-3324.

(46) Mesele, O. O.; Thompson, W. H. Removing the barrier to the calculation of activation energies. J. Chem. Phys. 2016, 145, 134107.

(47) Piskulich, Z. A.; Mesele, O. O.; Thompson, W. H. Removing the barrier to the calculation of activation energies: Diffusion coefficients and reorientation times in liquid water. J. Chem. Phys. 2017, 147, 134103-6.

(48) Piskulich, Z. A.; Mesele, O. O.; Thompson, W. H. Activation energies and beyond. J. Phys. Chem. A 2019, 123, 71857194.

(49) Piskulich, Z. A.; Thompson, W. H. The dynamics of supercooled water can be predicted from room temperature simulations. J. Chem. Phys. 2020, 152, 074505. 
(50) Price, W. S.; Ide, H.; Arata, Y. Selfdiffusion of supercooled water to $238 \mathrm{~K}$ using PGSE NMR diffusion measurements. J. Phys. Chem. A 1999, 103, 448-450.

(51) Xu, Y.; Petrik, N. G.; Smith, R. S.; Kay, B. D.; Kimmel, G. A. Growth rate of crystalline ice and the diffusivity of supercooled water from 126 to 262 K. Proc. Natl. Acad. Sci. USA 2016, 113, 1492114925.

(52) Piskulich, Z. A.; Thompson, W. H. On the temperature dependence of liquid structure. J. Chem. Phys. 2020, 152, 011102.

(53) Mendis, C. H.; Piskulich, Z. A.; Thompson, W. H. Tests of the Stokes-Einstein relation through the shear viscosity activation energy of water. J. Phys. Chem. B 2019, 123, 5857-5865.

(54) Tolman, R. C. Statistical mechanics applied to chemical kinetics. J. Am. Chem. Soc. 1920, 42, 2506-2528.

(55) Truhlar, D. G. Interpretation of the activation energy. J. Chem. Educ. 1978, 55, 309-311.

(56) Levine, R. D.; Bernstein, R. B. Molecular Reaction Dynamics and Chemical Reactivity; Oxford University Press: New York, 1987.

(57) Berendsen, H. J. C.; Grigera, J. R.; Straatsma, T. P. The missing term in effective pair potentials. J. Phys. Chem. 1987, 91, 6269-6271.

(58) Joung, I. S.; Cheatham III, T. E. Determination of alkali and halide monovalent ion parameters for use in explicitly solvated biomolecular simulations. J. Phys. Chem. B 2008, 112, 9020-9041.

(59) The LAMMPS molecular dynamics package, http://lammps.sandia.gov.

(60) Plimpton, S. Fast parallel algorithms for short-range molecular dynamics. J. Comput. Phys. 1995, 117, 1-19.
(61) Lorentz, H. Ueber die anwendung des satzes vom virial in der kinetischen theorie der gase. Annalen der Physik 1881, 248, 127-136.

(62) Berthelot, D. Si la fonction doit etre continue par rapport a l'ensemble (xy), elle doit etre constante sur les characteristiques. Academie des Sciences 1898, 126, 1703-1855.

(63) Yeh, I.-C.; Hummer, G. System-size dependence of diffusion coefficients and viscosities from molecular dynamics simulations with periodic boundary conditions. J. Phys. Chem. B 2004, 108, 1587315879 .

(64) Shoemaker, D. P.; Garland, C. W.; Nibler, J. W. Experiments in Physical Chemistry; McGraw-Hill: New York, 1989 .

(65) Cieplak, P.; Kollman, P. Monte Carlo simulation of aqueous solutions of $\mathrm{Li}^{+}$and $\mathrm{Na}^{+}$using many-body potentials. Coordination numbers, ion solvation enthalpies, and the relative free energy of solvation. J. Chem. Phys. 2005, 123, 094506.

(66) Heuft, J. M.; Meijer, E. J. Density functional theory based molecular-dynamics study of aqueous chloride solvation. $J$. Chem. Phys. 2003, 119, 11788-11791.

(67) Raugei, S.; Klein, M. L. An ab initio study of water molecules in the bromide ion solvation shell. J. Chem. Phys. 2002, 116, 196-202.

(68) Heuft, J. M.; Meijer, E. J. Density functional theory based molecular-dynamics study of aqueous iodide solvation. $J$. Chem. Phys. 2005, 92, 6761-6767.

(69) Boisson, J.; Stirnemann, G.; Laage, D.; Hynes, J. T. Water reorientation dynamics in the first hydration shells of $\mathrm{F}^{-}$and $\mathrm{I}^{-}$. Phys. Chem. Chem. Phys. 2011, 13, 19895. 
(70) Koneshan, S.; Rasaiah, J. C.; LyndenBell, R. M.; Lee, S. H. Solvent structure, dynamics, and ion mobility in aqueous solutions at $25^{\circ} \mathrm{C}$. J. Phys. Chem. B 1998, 102, 4193-4204.

(71) Koneshan, S.; Rasaiah, J. C.; Dang, L. X. Computer simulation studies of aqueous solutions at ambient and supercritical conditions using effective pair potential and polarizable potential models for water. $J$. Chem. Phys. 2001, 114, 7544-7555.

\section{TOC Graphic}

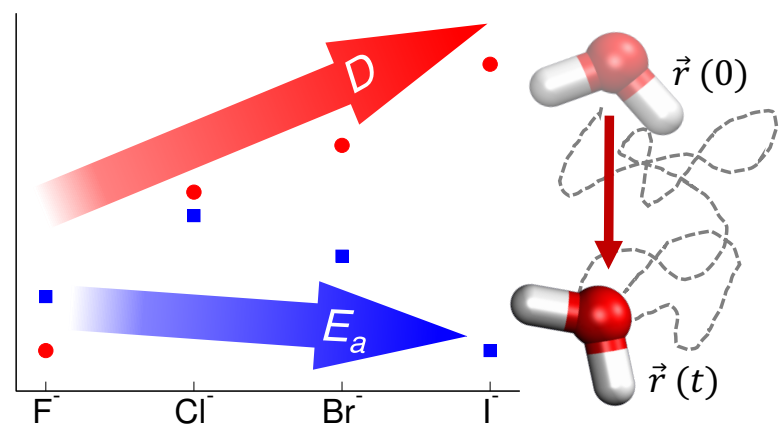

